\title{
Epileptic Seizure Prediction from Spectral, Temporal, and Spatial Features of EEG Signals Using Deep Learning Algorithms
}

\section{Nazanin Mohammadkhani Ghiasvand, Foad Ghaderi*}

\section{Human-Computer Interaction Lab, Faculty of Electrical and Computer Engineering, Tarbiat Modares University, Tehran, Iran}

\section{ABSTRACT}

Introduction: Epilepsy is one of the most common brain disorders that greatly affect patients' life. However, early detection of seizure attacks can significantly improve their quality of life. In this study, we evaluated a deep neural network to learn robust features from electroencephalography (EEG) signals to automatically detect and predict seizure attacks. Materials and Methods: The architecture consists of convolutional neural networks and long short-term memory networks. It is designed to simultaneously capture spectral, temporal, and spatial information. Moreover, the architecture does not rely on explicit channel selection algorithms. The method is applied to the Children's Hospital of Boston-Massachusetts Ins titute of Technology dataset (CHB-MIT). To evaluate the method, the proposed model is trained in the patient-specific approach. Results: The proposed architecture achieves a sensitivity of $90.7 \pm 7.9$ percent, a false prediction rate of $0.12 / \mathrm{h}$, and a mean prediction time of 36.8 minutes. Moreover, in the cases of focal seizures, the proposed model estimates the seizure focus. Conclusion: The proposed model achieved a high capability in seizure prediction. Moreover, by using the automated feature selection of the deep learning algorithm, the patterns of the pre-ictal period in EEG signals were determined. Furthermore, by specifying the seizure focus, the model can help neurologists to take further curative actions.

*Corresponding Author: Foad Ghaderi

E-mail:fghaderi@modares.ac.ir

Keywords:

1. Patients

2. Deep Learning

3. Electroencephalography 


\section{"ييشبينى تشنج صرعى از روى ويزكَىهاى طيفى، زمانى و مكانى سيخنالهاى نوار مغزى با استفاده از الكوريتهمهاى يادكيرى عميق}

نازنين محمدخانى غياثوند، فؤاد قادرى"

آزمايشگاه تعامل انسان و كاميِيوتر، دانشكده مهندسى برق و كاميِوتر، دانشعاه تربيت مدرس، تهران، ايران

\section{اطلاعات مقاله:}

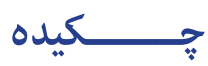

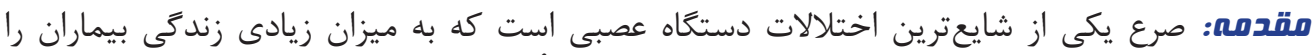

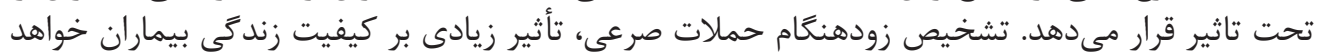

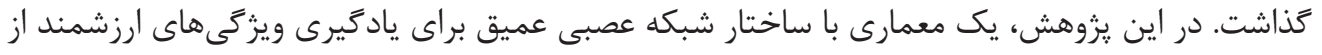

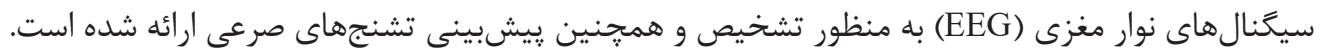

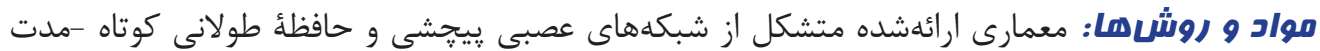

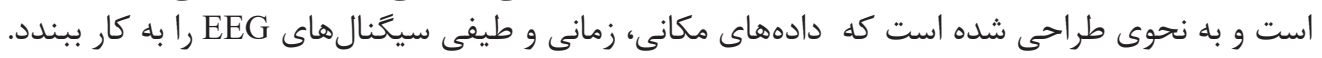

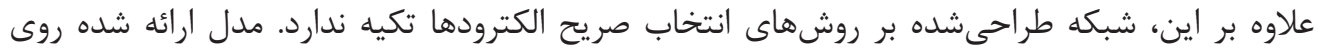

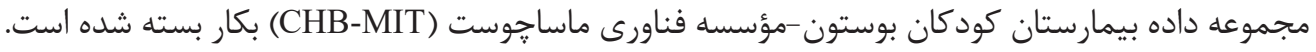

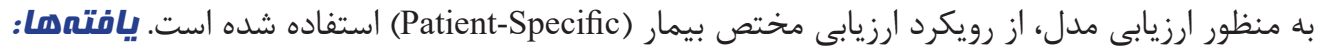

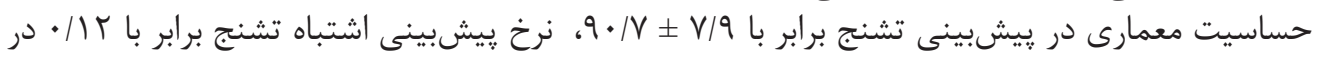

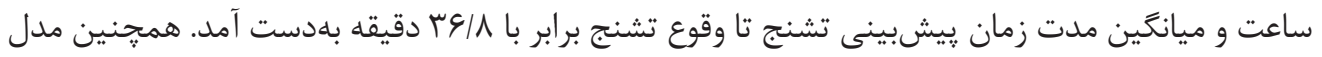

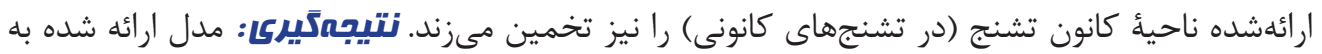

وازههاى كليدى:

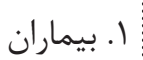

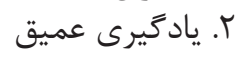

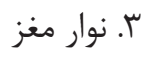

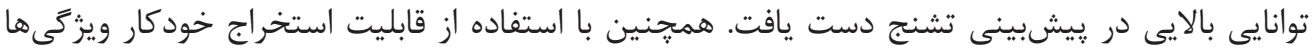

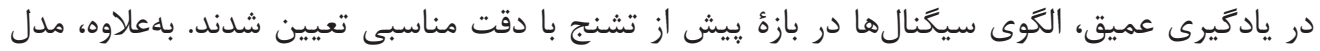

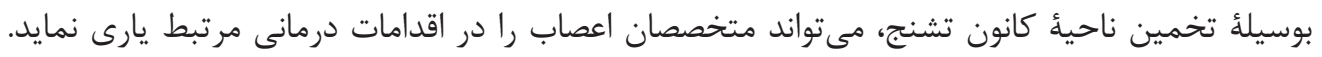




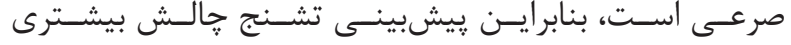

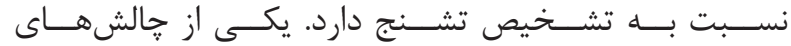

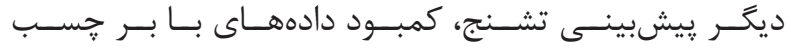

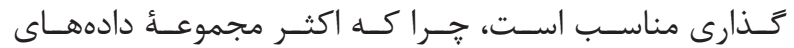

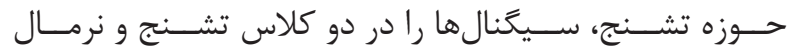

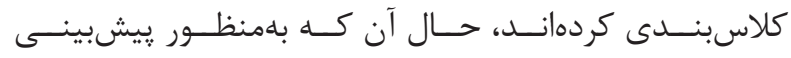

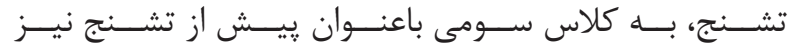

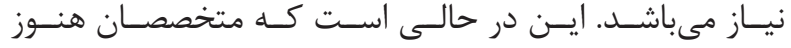

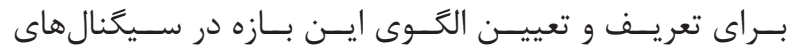

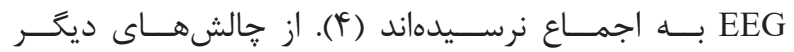

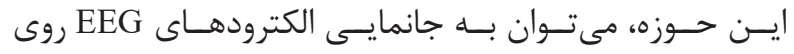

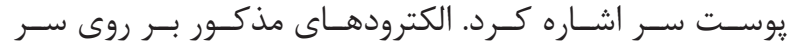

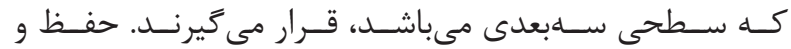

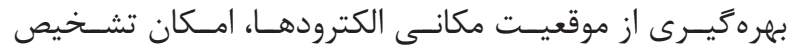

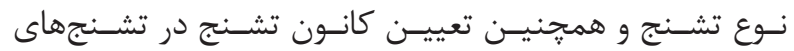

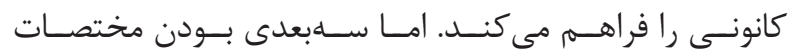

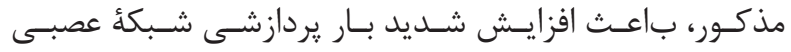

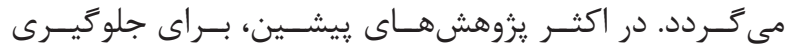

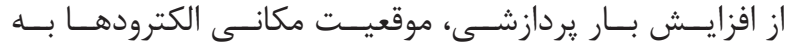

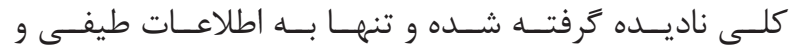

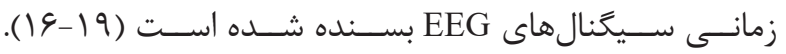

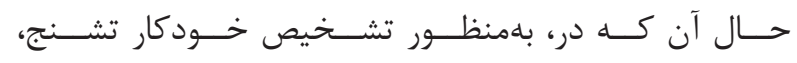

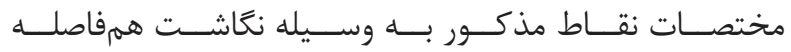

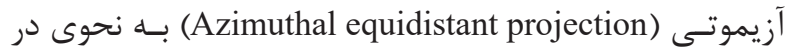

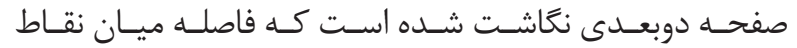

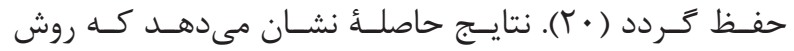

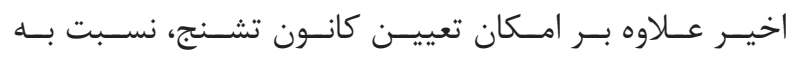

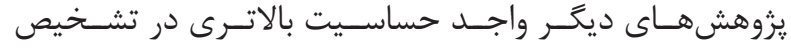

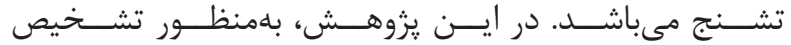

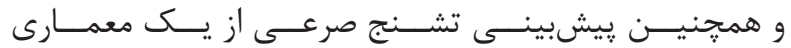

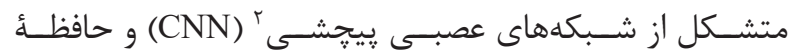

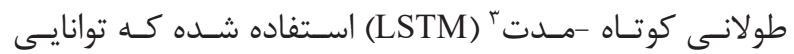

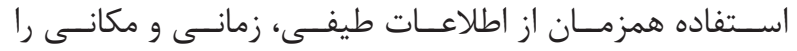

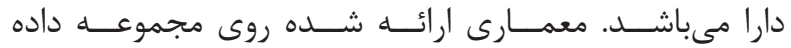

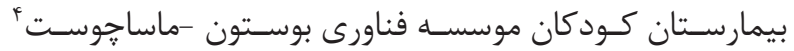

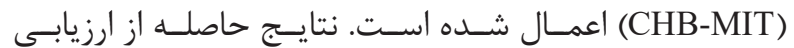

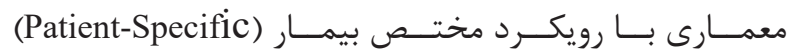

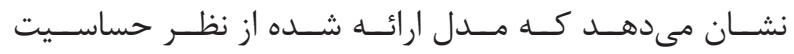

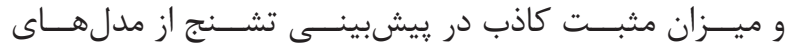

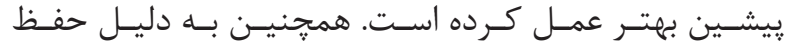

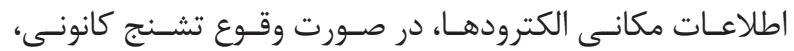

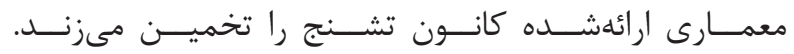

مواد و روشها

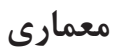

در ايــن يزوهــش، جهــت نيـلـل بـهـ دو هــدف تشــخيص و

${ }^{1}$ Electroencephalography; EEG

${ }^{2}$ Convolutional Neural Network

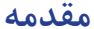

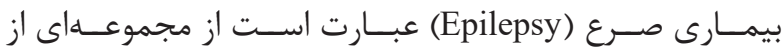

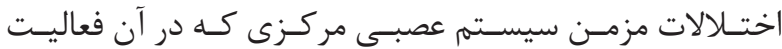

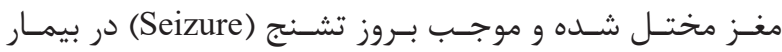

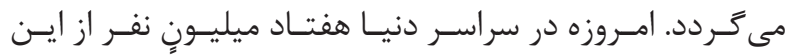

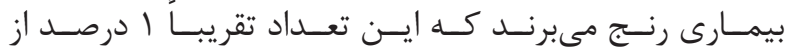

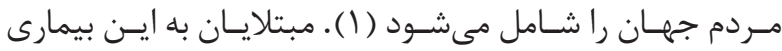

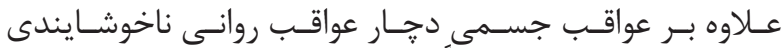

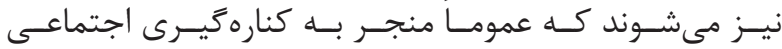

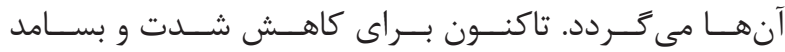

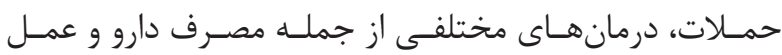

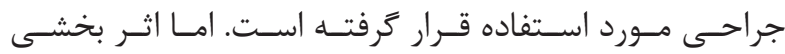

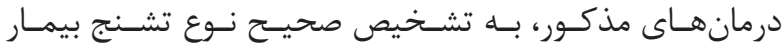

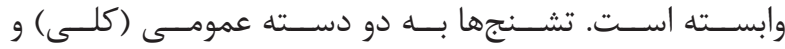

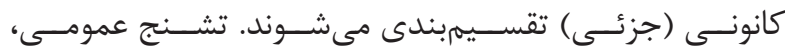

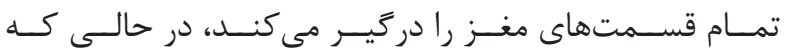

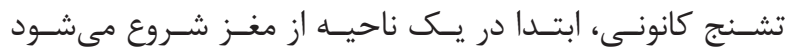

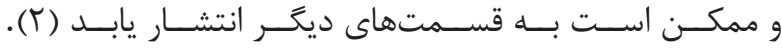

در صـورت شناسـايى صحيـح كانـون تشــنج در مغـز بيمـار،

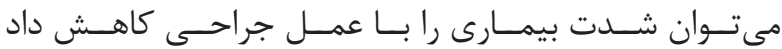

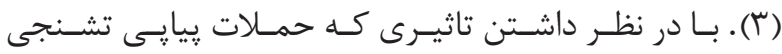

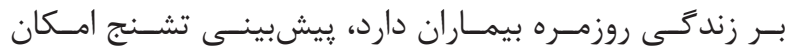

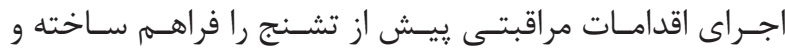

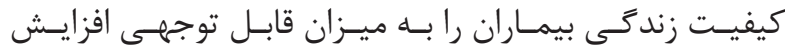

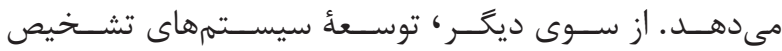

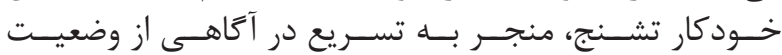

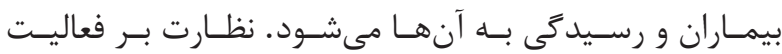

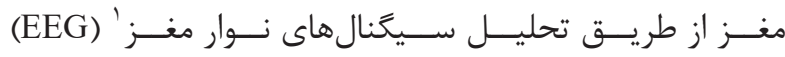

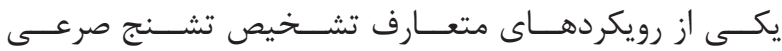

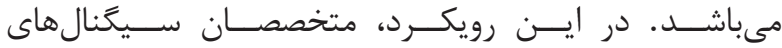
تالحل EEG

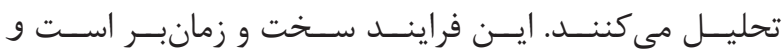

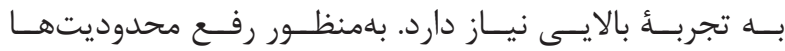

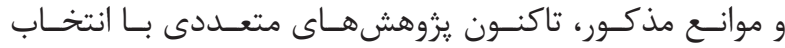

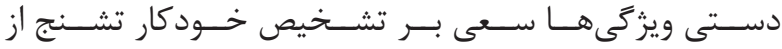

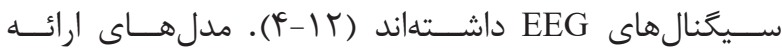

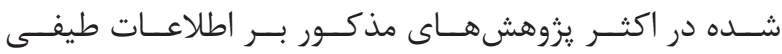

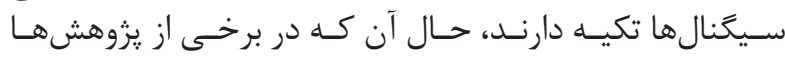

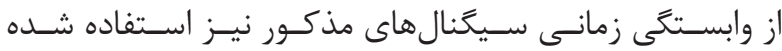

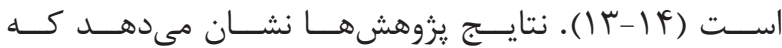

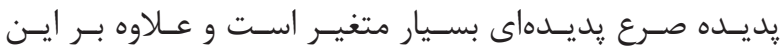

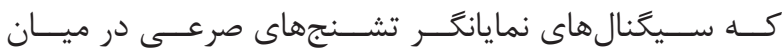

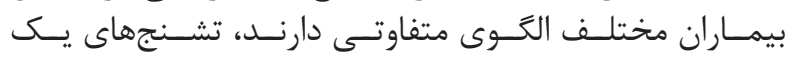

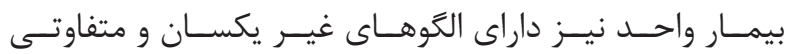

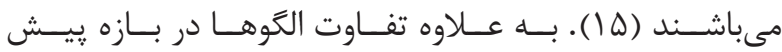

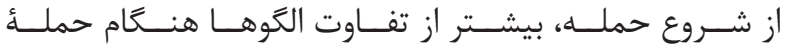

${ }^{3}$ Long Short Term Memory

${ }^{4}$ Childerns Hospital Boston Institute of Technology 


$$
\text { ييش پيردازش }
$$

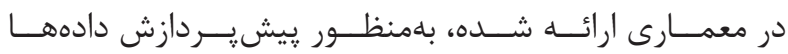

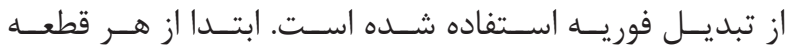

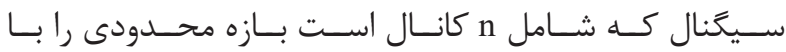

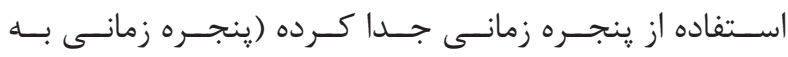

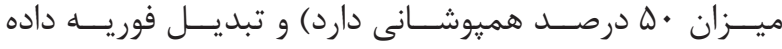
موجــود در هـــر كانـال را در ايسـن بــازه محاسـبه مى كنيـهم.

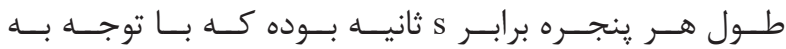

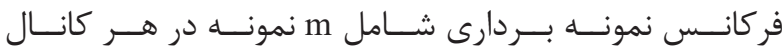

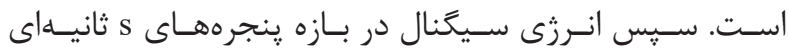

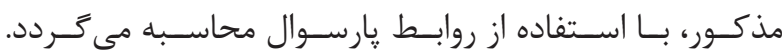

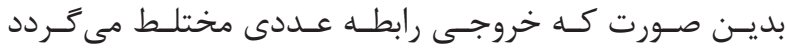

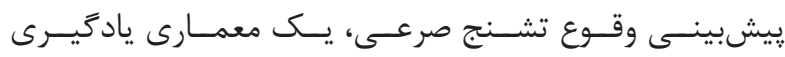

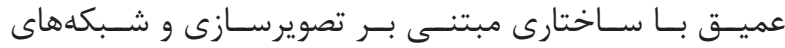

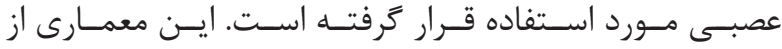

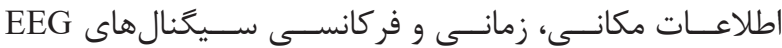

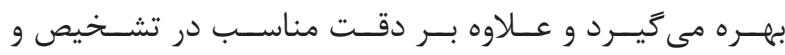

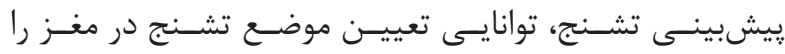

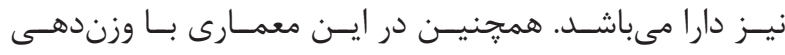

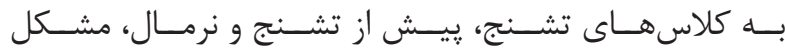

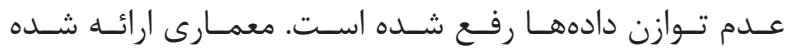

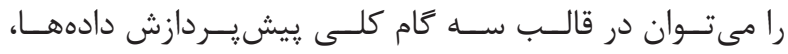

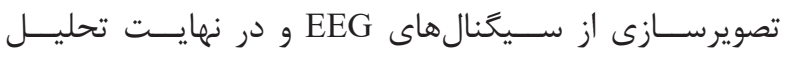

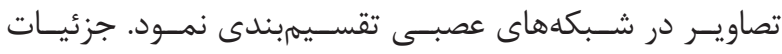

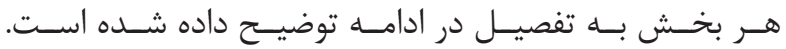

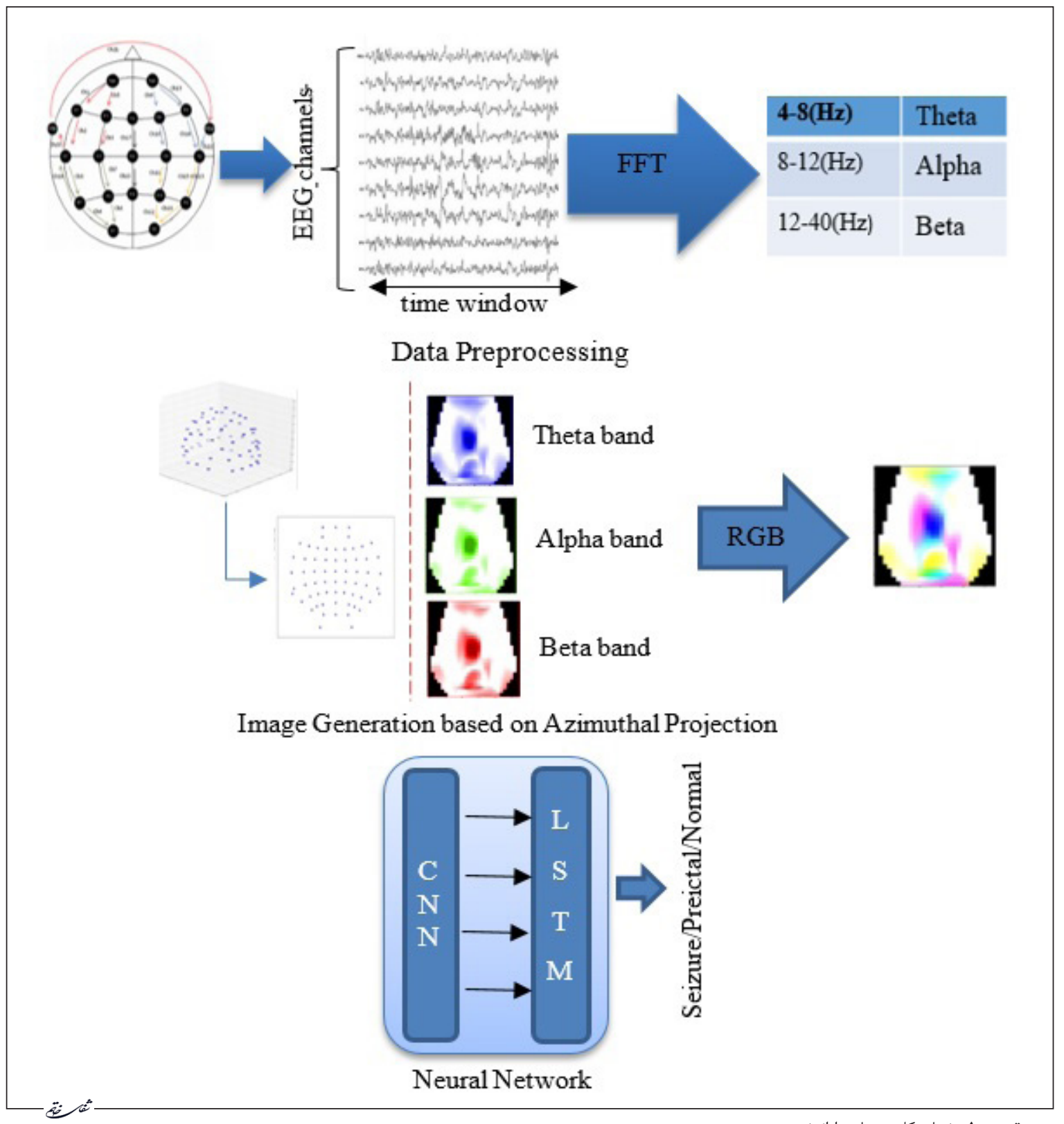

تصوير 1- شماى كلى معمارى ارائهشده 


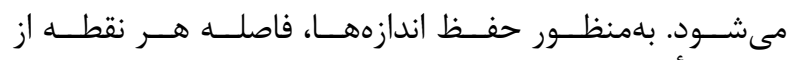

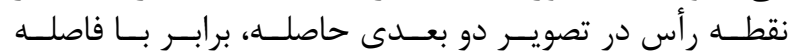

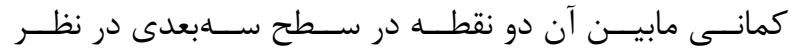

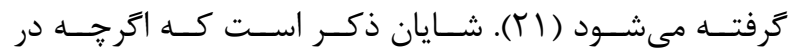

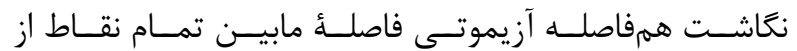

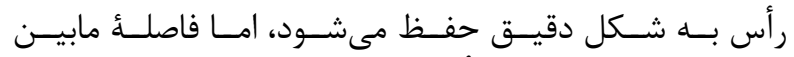

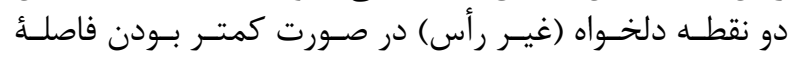

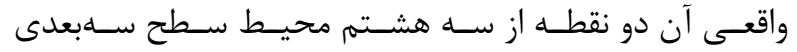

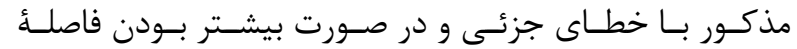

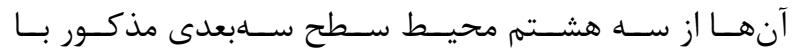

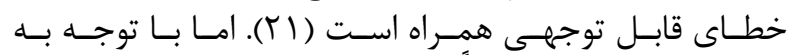

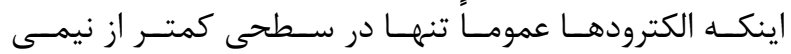

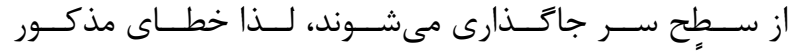

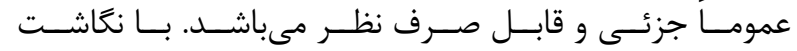

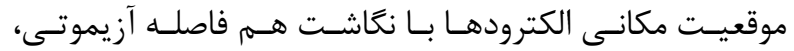

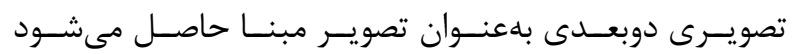

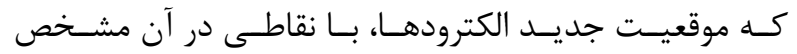

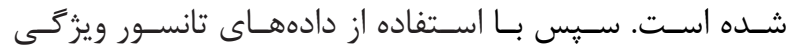

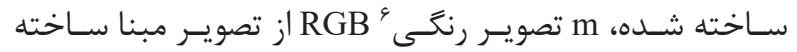

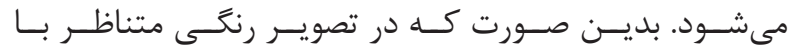

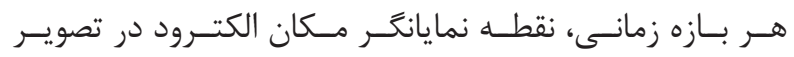

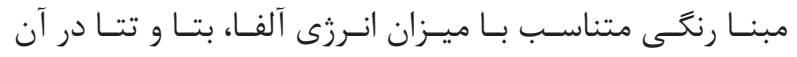

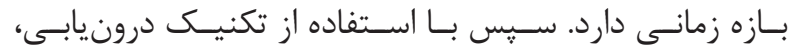

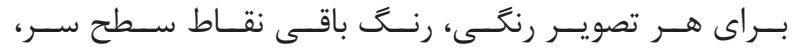

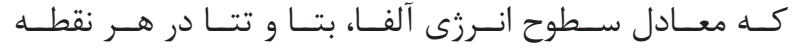

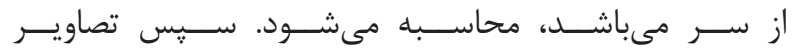

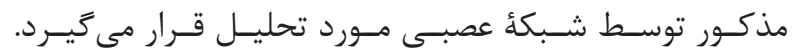

\section{شبكههاى عصبى ثيبششى}

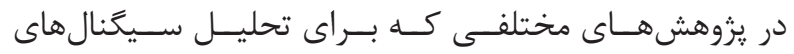

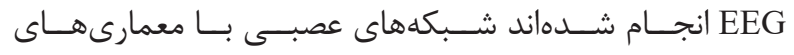

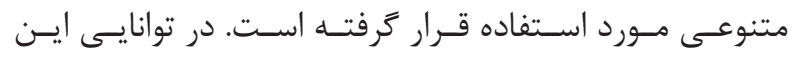

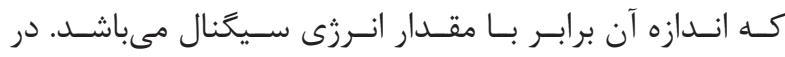

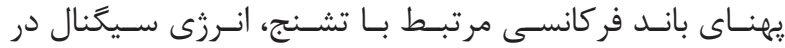

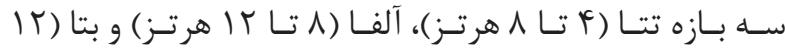

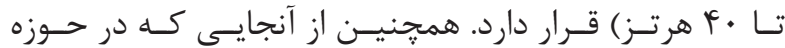

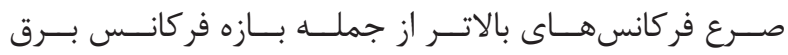

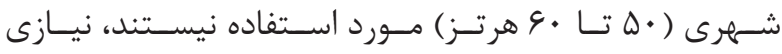

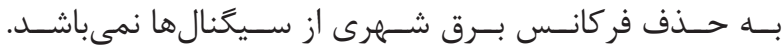

\section{تصويرسازى با سيخَنالهاى تEG}

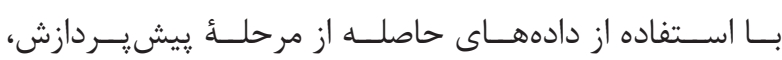

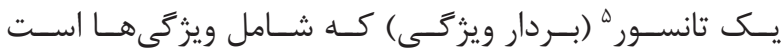

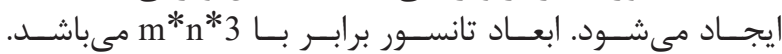

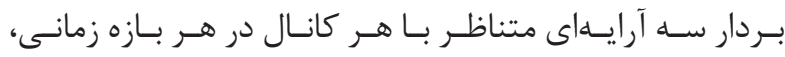

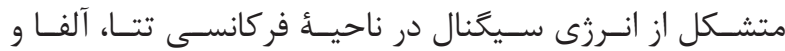

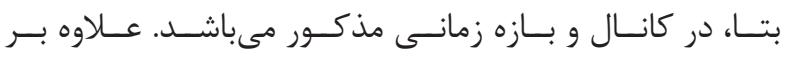

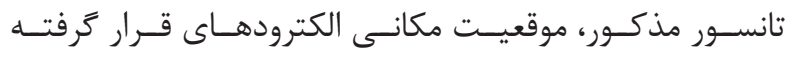

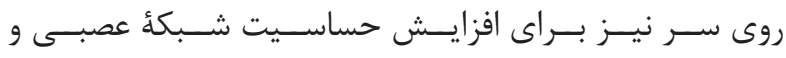

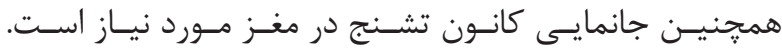

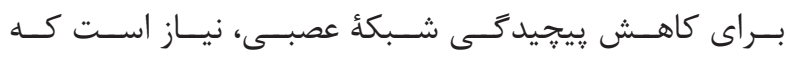

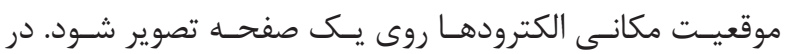

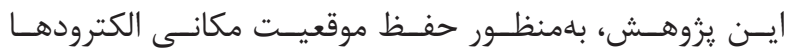

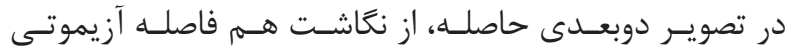

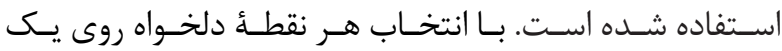

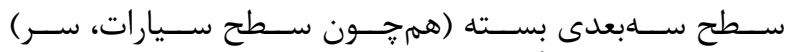

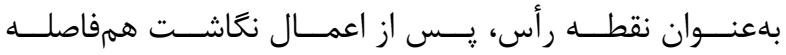

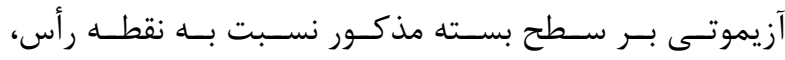

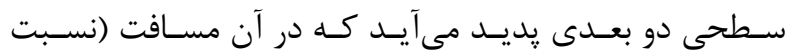

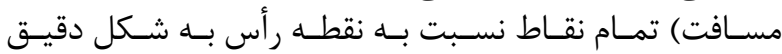

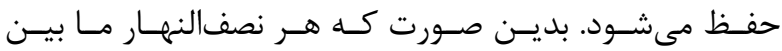

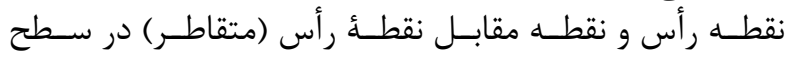

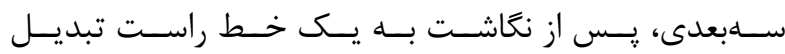

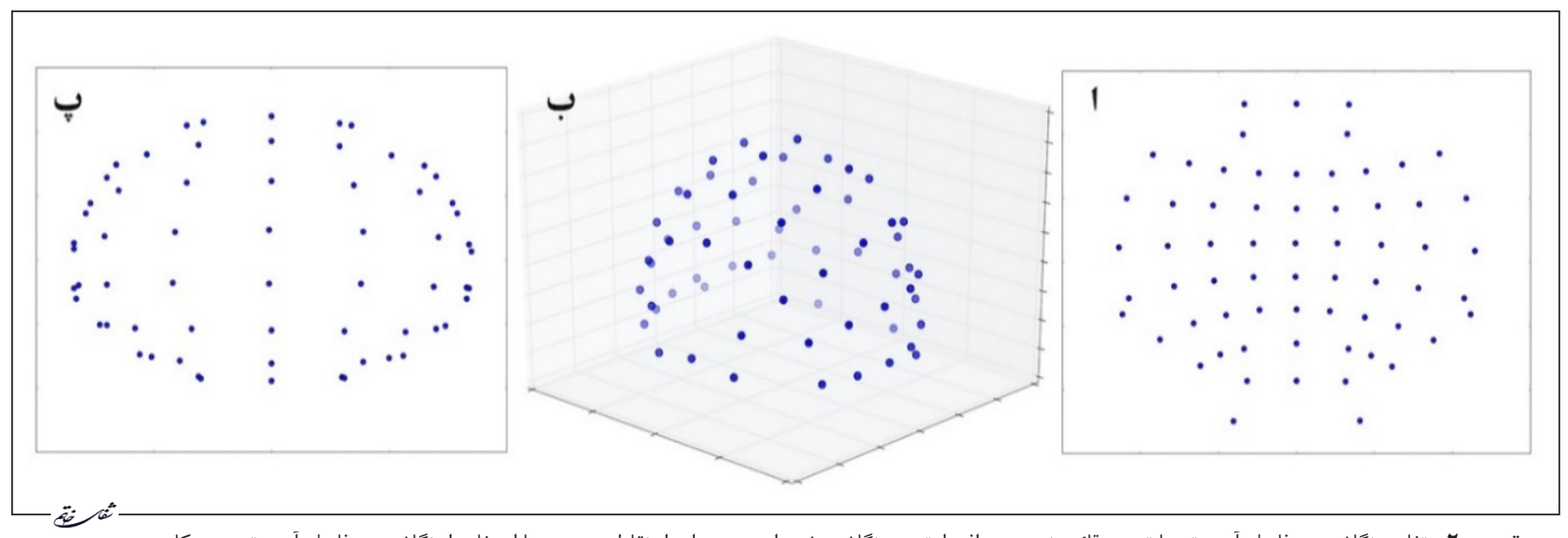

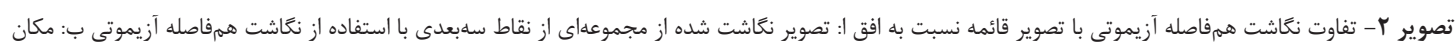

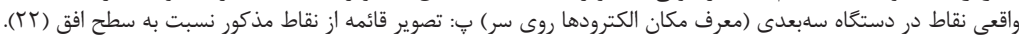




\section{وزندهى كلاسها}

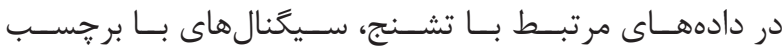

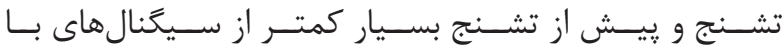

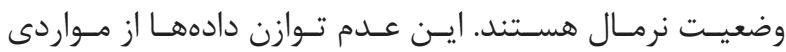

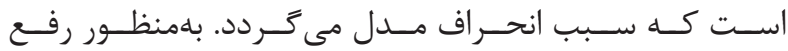

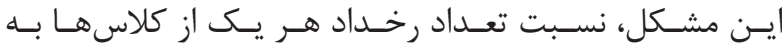

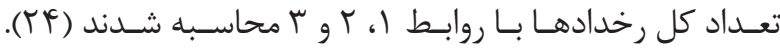

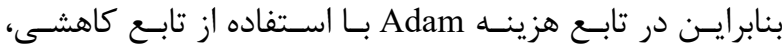

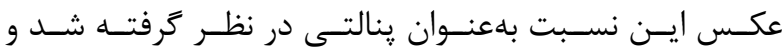

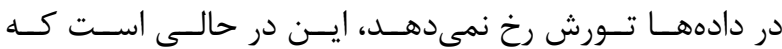

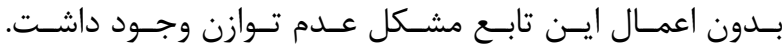
$W($ seizure $)=\frac{\text { Num(seizure_class) }}{\text { Num(all-class })}$

$W($ preictal $)=\frac{\text { Num(preictal_class })}{\text { Num(all-class })}$

$W($ normal $)=\frac{\text { Num(normal_class })}{\text { Num(all-class })}$

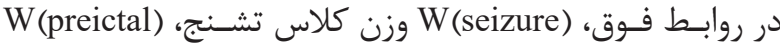

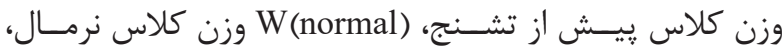
preictal-) تعـــــ Num (seizure_class)

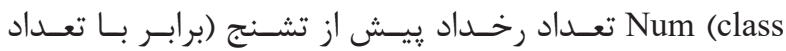

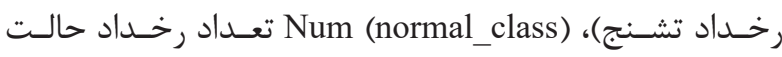

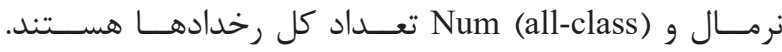

\section{ارزيابى معمارى زيشنههادى}

در ايـن يزثوهـش مجموعـهـ داده بيمارسـتان كـودكان بوسـتون

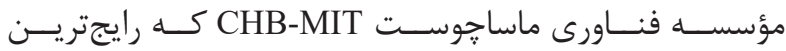

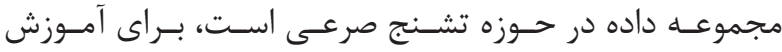

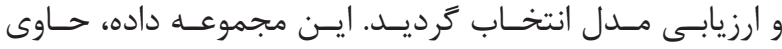

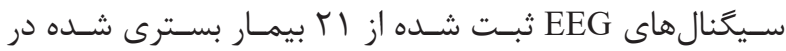

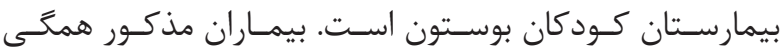

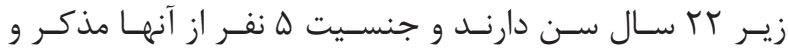

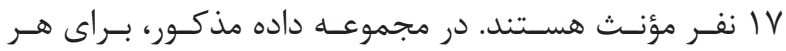

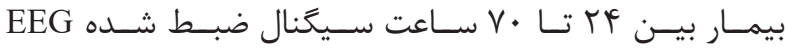

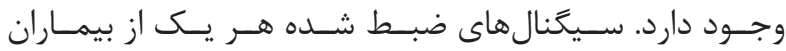

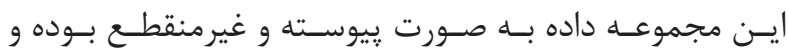

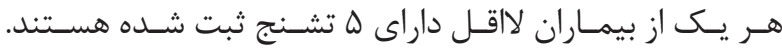

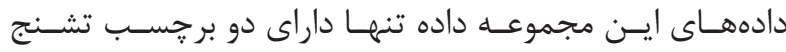

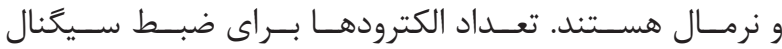

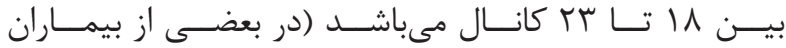

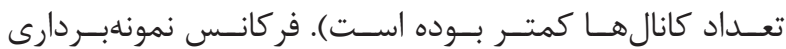

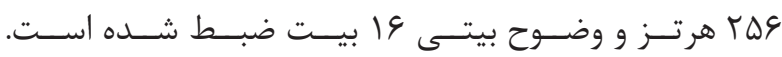

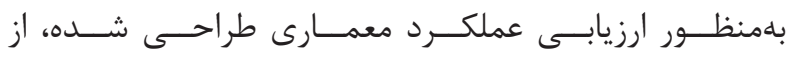

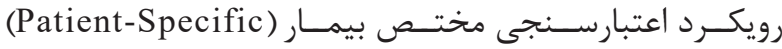

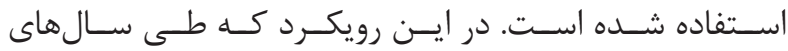

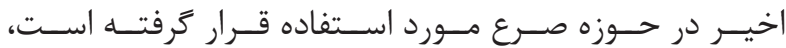

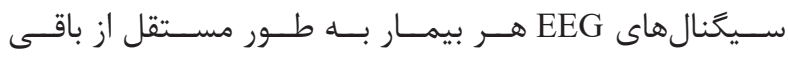

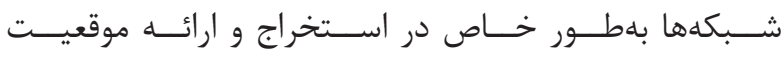

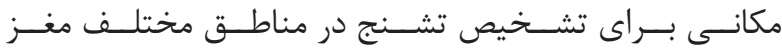

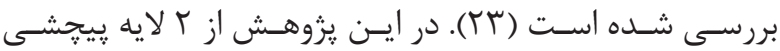

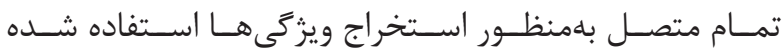

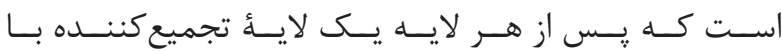

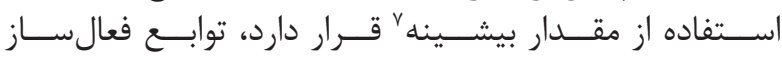

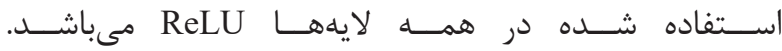

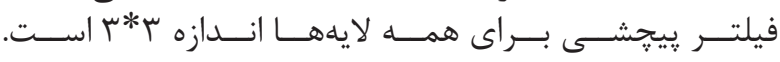

\section{شبكههاى عصبى بازگَشتى}

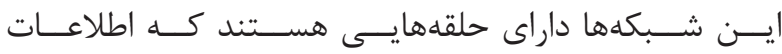

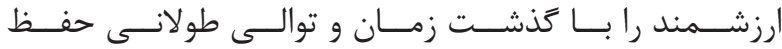

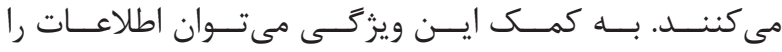

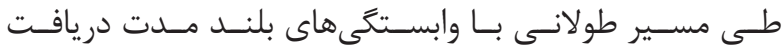

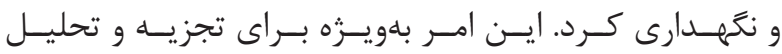

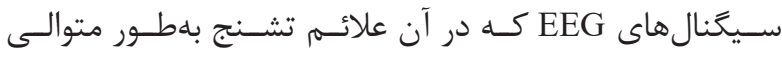

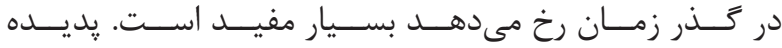

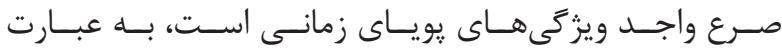

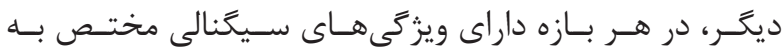

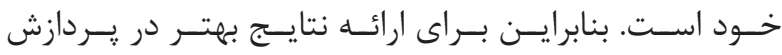

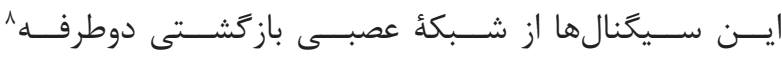

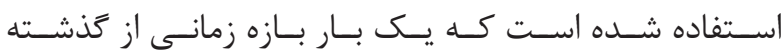

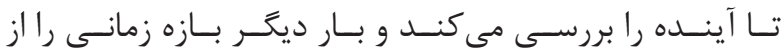

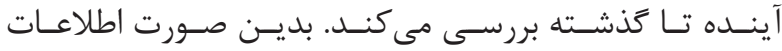

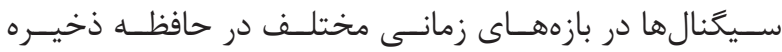

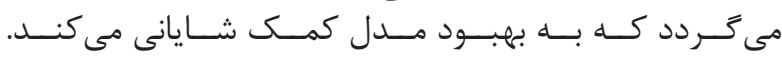

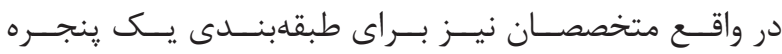

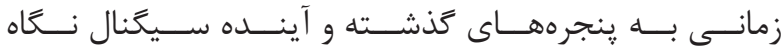

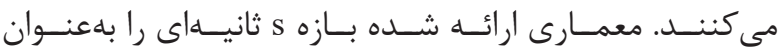

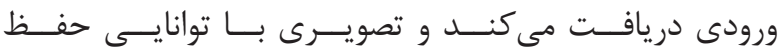

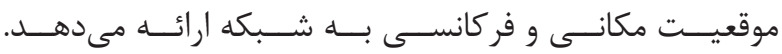

\section{رويكرد ييشبينى تشنج}

ســيخَنال هاى موجــود در مجموعـهـ داده CHB-MIT داراى دو

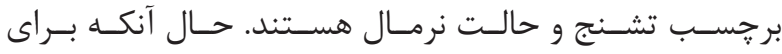

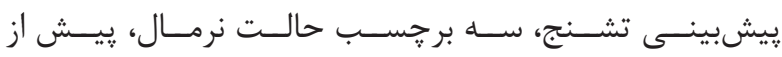

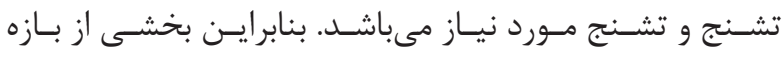

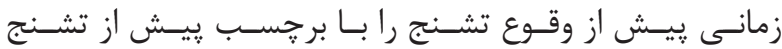

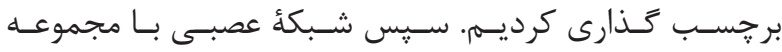

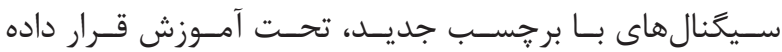

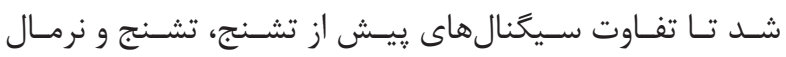

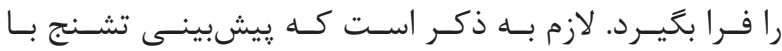

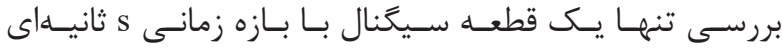

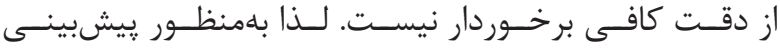

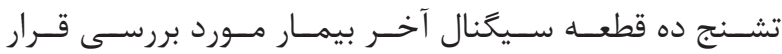

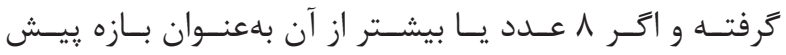

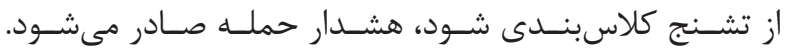

${ }^{7}$ Max pooling

${ }^{8}$ Bidirectional recurrent neural network 


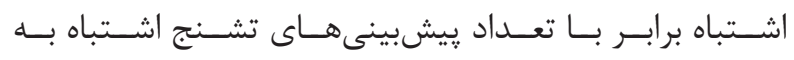

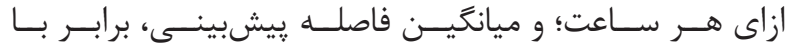

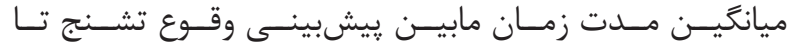

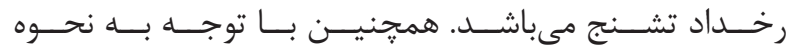

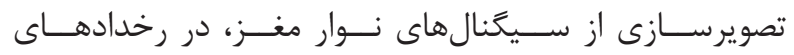

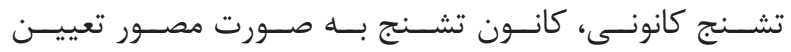

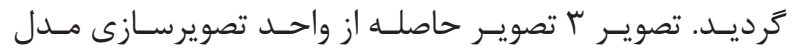

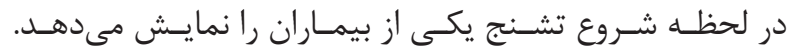

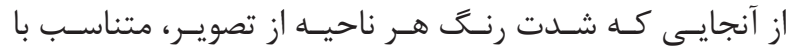

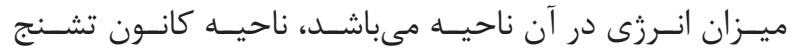

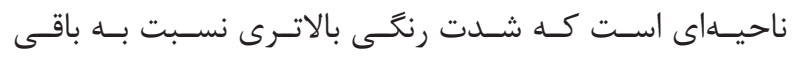

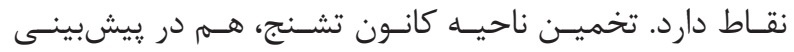

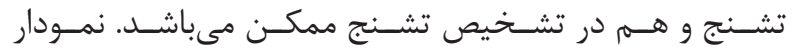

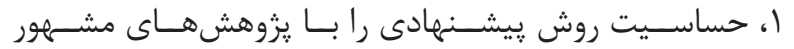

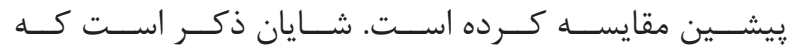

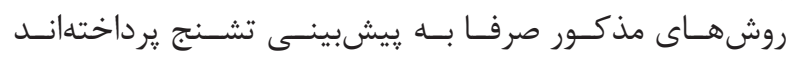

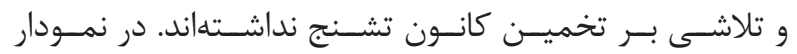

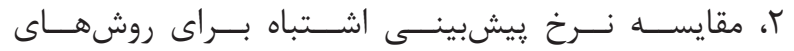

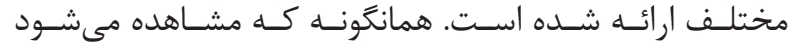

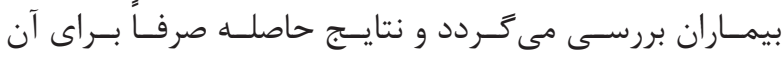

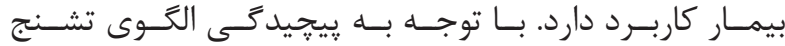

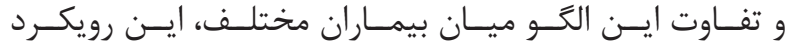

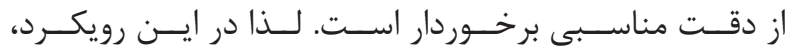

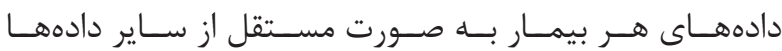

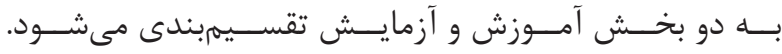
نكات عملى در زييادهسازى مدل

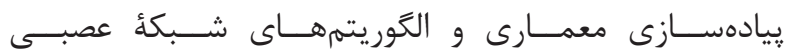

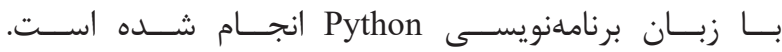

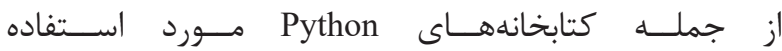

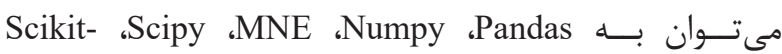
Tensorflow ،Keras ،Learn

بافتهها

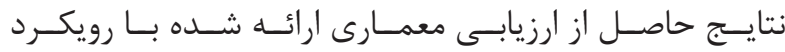

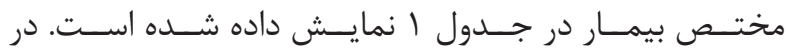

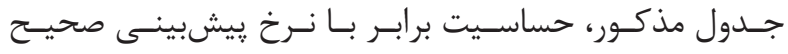

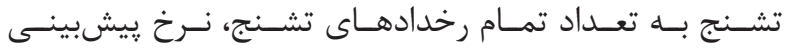

جدول ا- نتايج حاصل از ارزيابى معمارى با رويكرد مختص بيمار

\begin{tabular}{|c|c|c|c|}
\hline مياتخيث فاصلاد بيشينت (دقيقد) & ثرخ بيشىيثي اشتباه (ير ساعت) & حساسيت (درصد) & يبمارات \\
\hline$r \Delta / \Delta$ & $\cdot \gamma \cdot \lambda$ & 98 & بيماررا \\
\hline$r q / \cdot \Delta$ & $\cdot 1 \cdot 9$ & $9 \Delta$ & بيماربr \\
\hline rY/VT & $+1+$ & 99 & $r_{j}$ \\
\hline$r v / 9 \lambda$ & $\cdot 1 \cdot 9$ & QF & FيماررF \\
\hline IT/YF & $+1 *$ & $v \&$ & بيماره \\
\hline$F T / \cdot \Delta$ & $\cdot(1)$ & $\wedge \Delta$ & s, \\
\hline$F \Delta / \lambda$ & $\cdot 10 \mathrm{~V}$ & $\wedge \Lambda$ & $v_{j}$ \\
\hline Fr/A & $\cdot 1 \cdot 9$ & १९ & بيماررم \\
\hline$\Delta \Delta / T \Delta$ & $\cdot \gamma \cdot \lambda$ & qr & بيماروه \\
\hline Fr/Tr & $+1 \cdot 9$ & $9 \Delta$ & $1 \cdot$ \\
\hline YY/A9 & +10 & q4 & يبمارو \\
\hline Fr.rY & $.11 r$ & qF & بيماري \\
\hline FV/FS & $+1+$ & $9 \mathrm{~V}$ & بيمارو 19 \\
\hline Yq/FF & $+1+$ & $9 \Delta$ & بيمار •r \\
\hline FT/YS & $\cdot \beta \cdot \lambda$ & $१ \Delta$ & بيماران \\
\hline rSll & $.11 Y$ & $q . V r \pm V . q$. & مـياتخين \\
\hline
\end{tabular}



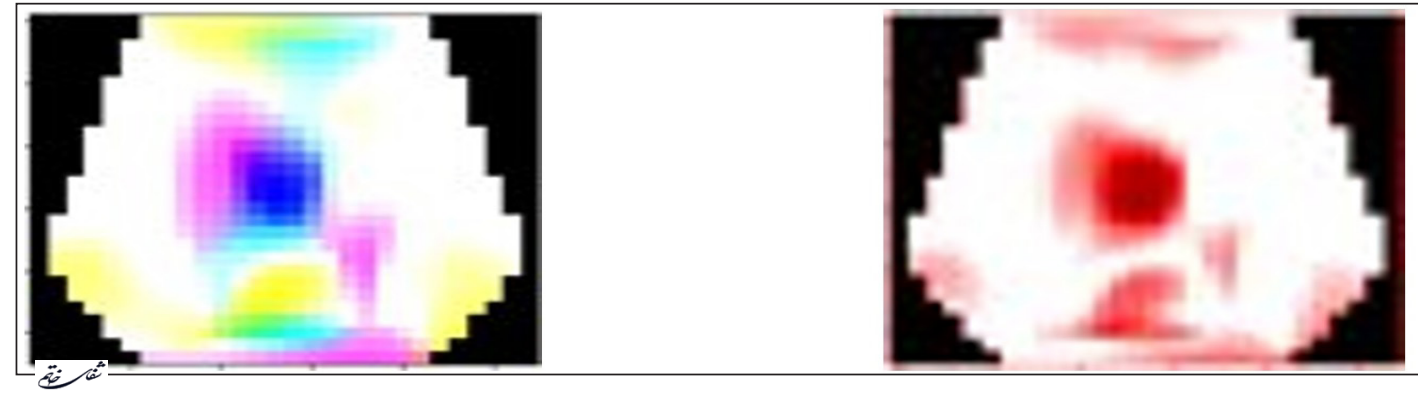

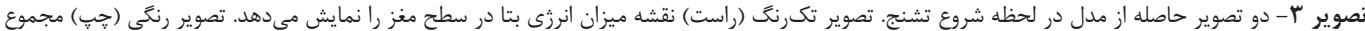

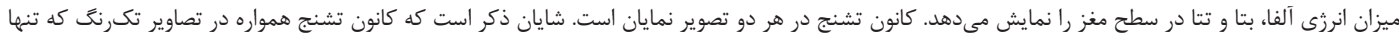

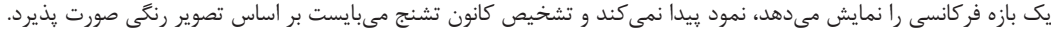

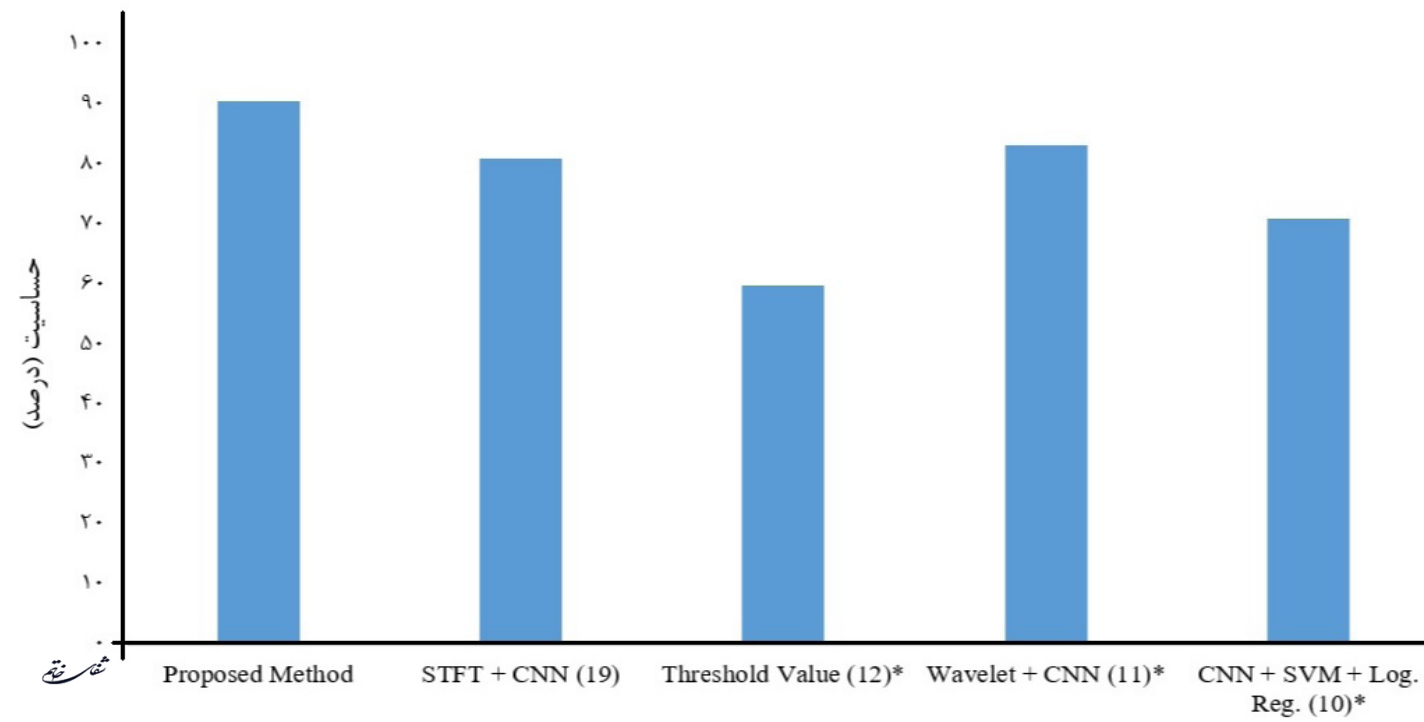

نمودار ا- مقايسه ميزان حساسيت روش ييشنهادى با روشهاى ييشين. خطاى محاسبهشده از نوع خطاى استاندارد مىباشد.:؛ خطاى استاندارد كزارش نشده است.

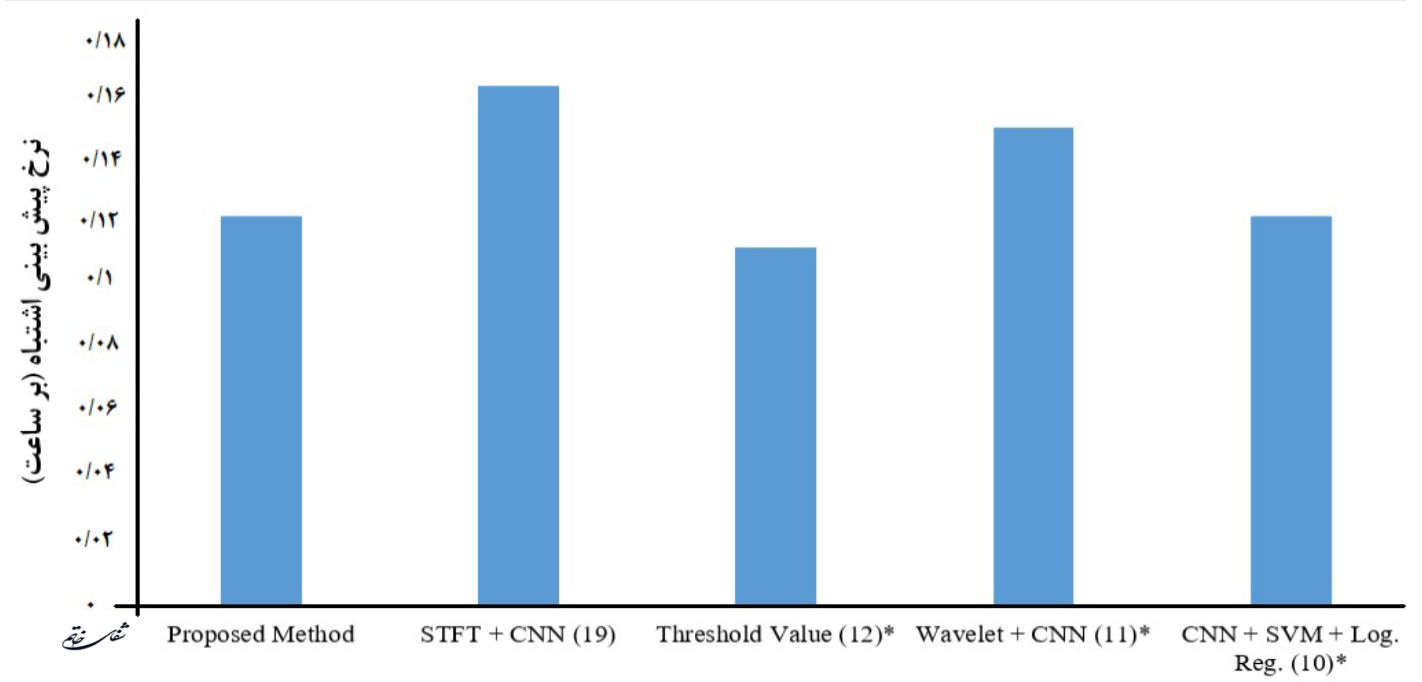

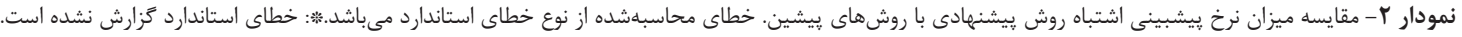

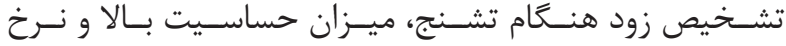

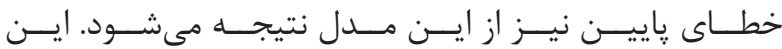

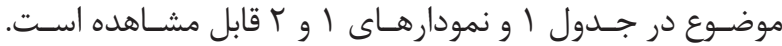

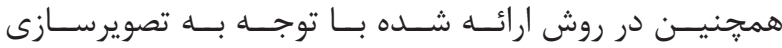

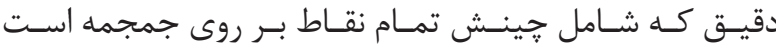

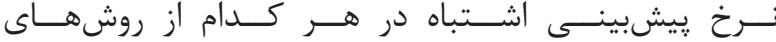

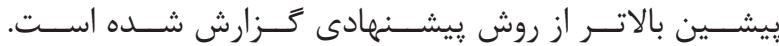
بحث و نتيجهل بيرى مزيست اصلـى مــدل ريشــنهادى ايـن اسـت كـهـ عـلاوه بــر 
توانايسى تخميـن ناحيـه وقـوع تشـنج در مغـز را فراهـم كـرده

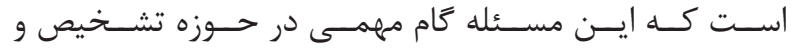

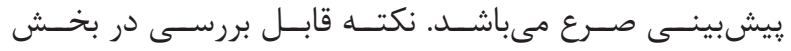

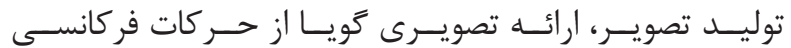

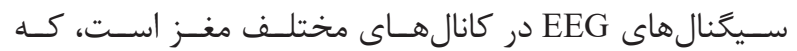

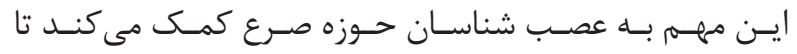

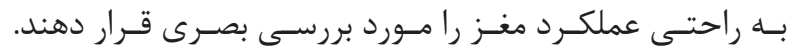

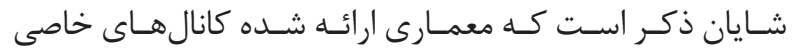

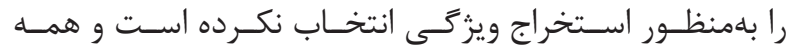

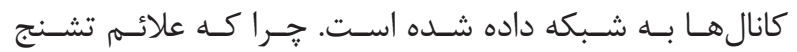

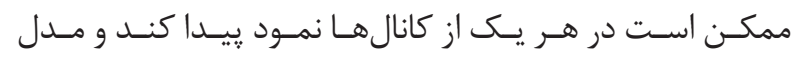

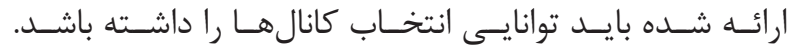

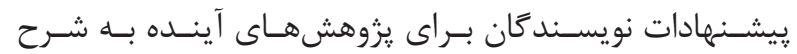

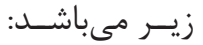

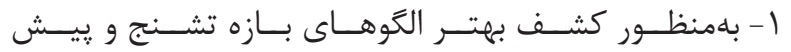

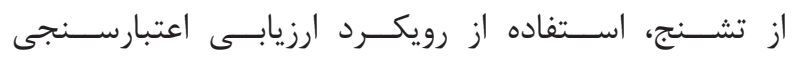

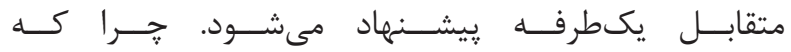

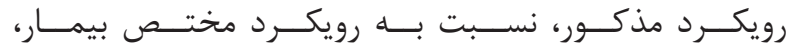

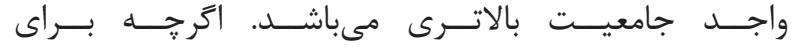

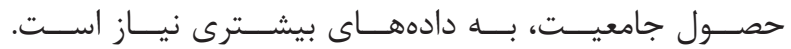

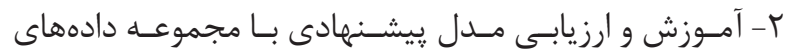

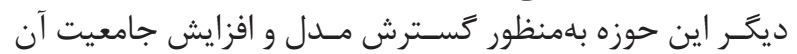

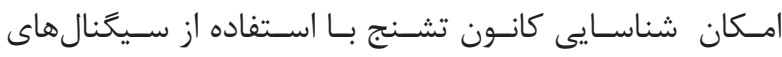

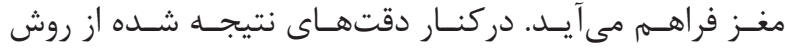

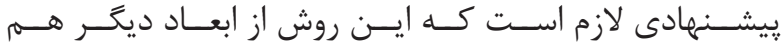

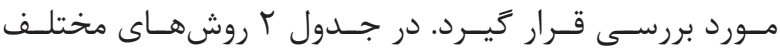

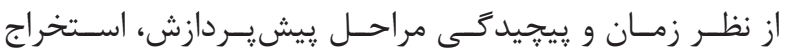

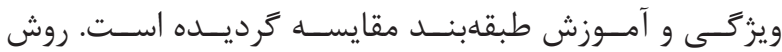
FFT +CNN+ BiLSTM

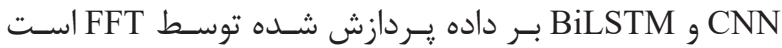

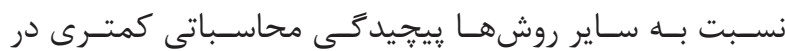

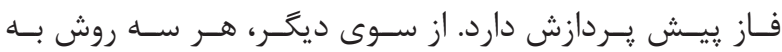

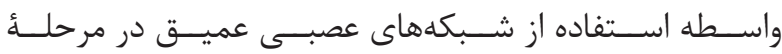

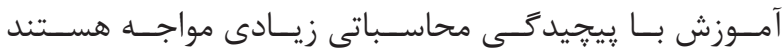

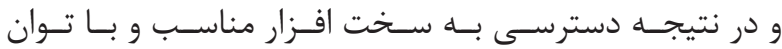

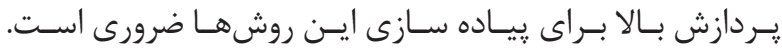

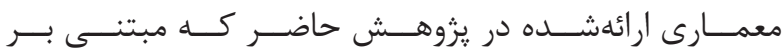

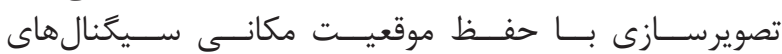

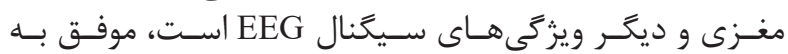

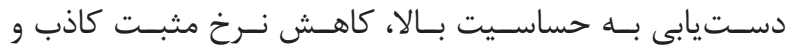

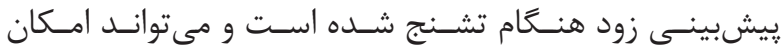

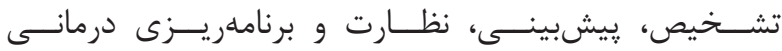

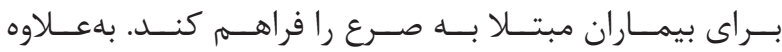

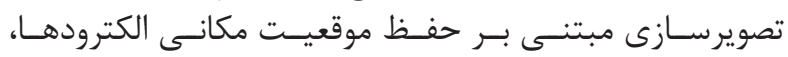

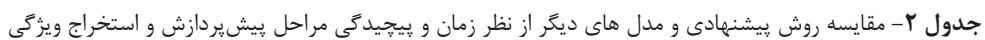

\begin{tabular}{|c|c|c|c|}
\hline بيجيدكى مر حلة آموزش & استخراج ويثَّى & بيش يردازش & 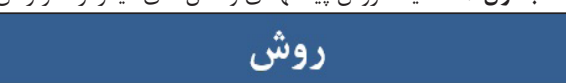 \\
\hline 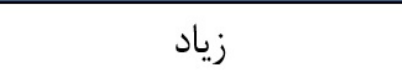 & سبك & متوسط & ساختار FFT +CNN+ BiLSTM \\
\hline زياد & سبك & زياد & ساختار STFT+ CNN+LSTM (19) \\
\hline زياد & سبك & زمان بر & ساختار CNN + تبديل موجك (11) \\
\hline
\end{tabular}

1. Thijs RD, Surges R, O’brien TJ. Sander JW, “Epilepsy in adults. vol. 393, ed: The Lancet, 2019; p. 611-716.

2. Stafstrom CE, Carmant L. Seizures and epilepsy: An overview for neuroscientists." Cold Spring Harb Perspect Med, 2015.

3. Miller JW, Hakimian S. Surgical treatment of epilepsy. ed: CONTINUUM Lifelong Learning in Neurology, 2013.

4. Sharif B, Jafari AH. Prediction of epileptic seizures from EEG using analysis of ictal rules on Poincaré plane," Computer Methods and Programs in Biomedicine. 2017; 11-22.

5. Aarabi A and He B. Seizure prediction in patients with focal hippocampal epilepsy. Clinical Neurophysiology, 2017; 7: 1299-307.
منابع

6. Eftekhar A, Juffali W, El-Imad J, Constandinou TG, Toumazou C. Ngram-derived pattern recognition for the detection and prediction of epileptic seizures. PLoS ONE, 2014; 6 .

7. Park Y, Luo L, Parhi KK, Netoff T.Seizure prediction with spectral power of EEG using cost-sensitive support vector machines. Epilepsia, 2011; 10: 1761-70.

8. Gadhoumi K, Lina JM, Gotman J. Seizure prediction in patients with mesial temporal lobe epilepsy using EEG measures of state similarity. Clinical Neurophysiology, 2013; 9: 1745-754.

9. Rogowski Z, Gath I, Bental E. On the prediction of epileptic seizures. Biological Cybernetics, 1981; 1: 9-15

10. Mirowski P, Madhavan D, Lecun Y, Kuzniecky R. 
Classification of patterns of EEG synchronization for seizure prediction. Clinical Neurophysiology, 2009; 11: 1927-940.

11. Beniczky S, Aurlien H, Brøgger JC, FuglsangFrederiksen A, Martins-Da-Silva A, Trinka E et al. Standardized Computer-Based Organized Reporting of EEG: SCORE. Epilepsia, 2013; 6: 1112-24.

12. Winterhalder M, Schelter B, Maiwald T, Brandt A, Schad A, Schulze-Bonhage A et al. Spatio-temporal patient-individual assessment of synchronization changes for epileptic seizure prediction. Clinical Neurophysiology, 2006; 11: 2399-413.

13. Alexandros T, Markos G, Dimitrios G, Evaggelos C, Astrakas L, Konitsiotis S et al. Automated Epileptic Seizure Detection Methods: A Review Study. in Epilepsy -Histological, Electroencephalographic and Psychological Aspects: InTech, 2012.

14. Shoeb AH. Application of machine learning to epileptic seizure onset detection and treatment. ed: Massachusetts Institute of Technology, 2009.

15. Panayiotopoulos CP. A Clinical Guide to Epileptic Syndromes and their Treatment. Springer-Verlag London, 2010.

16. Zhang $\mathrm{Z}$ and Parhi KK. Low-Complexity Seizure Prediction From iEEG/sEEG Using Spectral Power and Ratios of Spectral Power. IEEE Transactions on Biomedical Circuits and Systems, 2016; 3: 693-706

17. Alickovic E, Kevric J, Subasi A. Performance evaluation of empirical mode decomposition, discrete wavelet transform, and wavelet packed decomposition for automated epileptic seizure detection and prediction," Biomedical Signal Processing and Control. 2018; 94-102.

18. Khan H, Marcuse L, Fields M, Swann K, Yener B. Focal onset seizure prediction using convolutional networks. IEEE Transactions on Biomedical Engineering, 2018; 9: 2109-118.

19. Truong ND, Nguyen AD, Kuhlmann L, Bonyadi MR, Yang J, Ippolito S et al. Convolutional neural networks for seizure prediction using intracranial and scalp electroencephalogram. IEEE Transactions on Biomedical Engineering. 2018; 104-111.

20. Thodoroff P, Pineau J, Lim A. Learning Robust Features using Deep Learning for Automatic Seizure Detection. Journal of Machine Learning Research, 2016.

21. Snyder JP. Map projections- a working manual in Geological Survey professional paper; 1395," U.S. Geological Survey professional paper 1395, 1987. [Online]. Available: http://pubs.usgs.gov/pp/1395/report. pdf

22. Bashivan P, Rish I, Yeasin M, Codella N. Learning Representations from EEG with Deep RecurrentConvolutional Neural Networks," presented at the ICLR 2016.

23. Guo N, Yang Z, Jia Y, Wang L. Model updating using correlation analysis of strain frequency response function. Mechanical Systems and Signal Processing, 2016; 284-99. 